\title{
Experimental study on Flow Field Characteristics of Microbursts
}

\author{
Ramesh D. K ${ }^{\# 1}$, Gangadhar K.A ${ }^{\# 2}$, Dhananjaya D.R.. ${ }^{\# 3}$ Gokul S..4 ${ }^{\# \text { Varunkumar S." }}$ \\ \# Mechanical Engineering, Bangalore University \\ University Visvesvaraya College of Engineering, Bangalore, Karnataka, India -560001 \\ ${ }^{1}$ rameshdkuvce@gmail.com \\ 2 engineer.gangadhar@gmail.com \\ 5aru.7133910@gmail.com
}

\begin{abstract}
Severe thunderstorm is an important weather phenomenon which impact aviation, space vehicle launch, agriculture in besides its damaging potential for life and properties. The most important occurrence in the thunderstorm is the downburst, which is made up of moderately rotating column of air which upon impacting on the ground tends to burst violently. It is believed that when the upward moving of hot buoyant air can no longer sustain subsides into downburst. The downburst creates an impact on the ground and flows radially outwards. Downbursts are categorized as either microburst or macroburst depending on horizontal extension of damage. In this work an effort has been put to simulate the dry microburst experimentally using the impinging jet model for investigating the micro flow dynamics and scale dependency of microburst flow. Flow is visualized using a smoke generator for understanding the flow dynamics.
\end{abstract}

Keyword - Downburst, Macroburst, Microburst, Virga, Vortices.

\section{INTRODUCTION}

Microburst is a natural phenomenon which causes intense downdraft upon impacting the ground and forming a damaging outflow with range less than $4 \mathrm{~km}$. They produce remarkable wind shear and extreme winds near ground with the wind profile that differs from the Atmospheric boundary layer. In early 1900's, the definition of microburst has been further specified by Federal Aviation Administration, as wind shear with peak to peak wind speed differences of more than $15 \mathrm{~m} / \mathrm{s}$ over distance less than $4.5 \mathrm{~km}$, for the implementation of Terminal Doppler Weather Radar and low level wind shear alert system at major airports. Due to its momentary nature, microburst usually have very small lifespan and large vertical velocity component, which makes it difficult to detect but can be studied by Doppler radar. Several field research has been conducted to study the origin flow structure and meteorological parameters of microburst. Research by Northern Illinois Meteorological Research on Downburst, Chicago and Joint Airport Weather Studies(JAWS), Denver collected a wide range of data on microburst occurring in nature.

The famous atmospheric scientist, Fujita (1981) observed and studied the flow due to downburst. He classified downburst as either microburst $(<4 \mathrm{~km})$ or macro burst $(>4 \mathrm{~km})$ on the basis of their horizontal extent of the damage and also characterized according to the level of precipitation, i.e. wet microburst (downburst occurs along with rain) and dry microburst which consists virga (sublimation before reaching the earth). One of the first physical model of a thunderstorm downburst was made by Fujita at the University of Chicago in the 1970's (Fujita, 1990). Fujita's laboratory model consists of a buoyantly impinging plume impacting a surface, with the front edge made visible by dry-ice smoke.

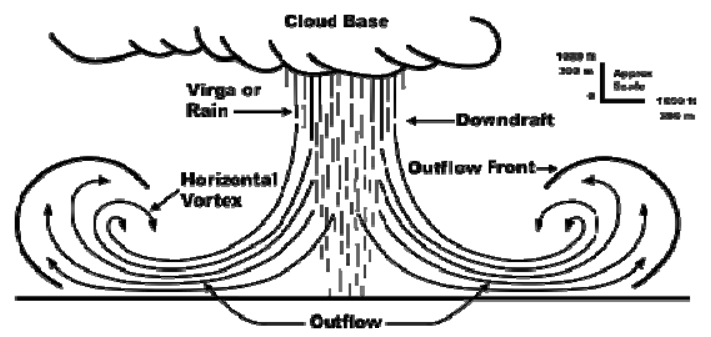

Fig.1. A typical flow field of microburst 


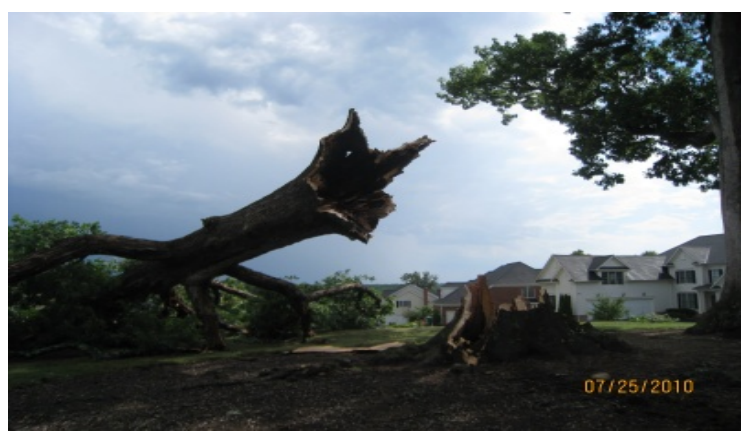

Fig.2. microburst effect in Northern Virginia

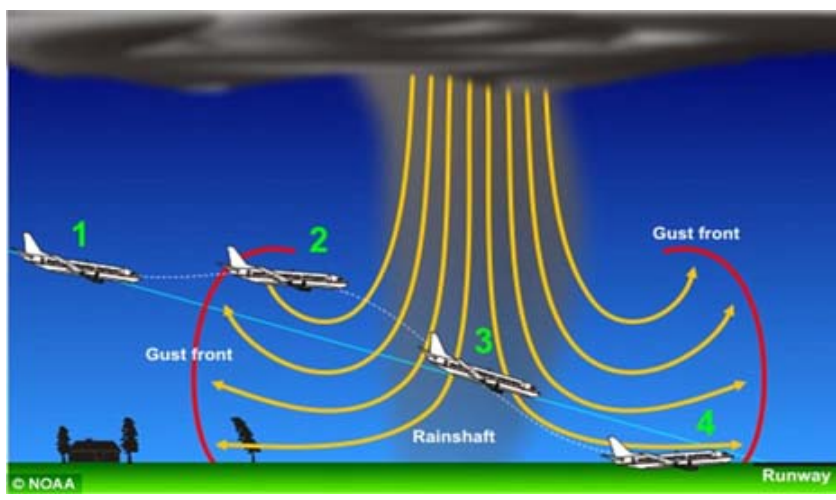

Fig.3. microburst loading effect on landing plane.

\section{Experimental Setup}

To simulate stationary axisymmetric dry microbursts physically, steady impinging jet model is considered. The investigation on microburst features are carried in an impinging jet model which is fabricated with an air blower of $1 \mathrm{~kW}$ used to generate impinging jet and an exit nozzle of one-inch diameter(D) is fixed at an intermediate portion of the model as shown in the Fig.4.

The jet is impinged on the base of the model made of wooden board, through a nozzle from two different heights and its velocity and pressure are measured radially at the required coordinates. The distance from the tip of the nozzle and the wooden platform can be varied to different heights i.e. Different H/D ratios taken as 1,2 . The velocity and pressure are an important parameter that is required to measure the degree of turbulence in the region of impingement.

The experimental readings were taken at two different H/D ratios with a jet velocity of $10 \mathrm{~m} / \mathrm{s}$. The magnitude of the velocity is measured from axis of impingement of jet at 10 different locations radially using anemometer which has a calibrated error of $3 \pm .02 \%$ and has measuring range of 1 to $25 \mathrm{~m} / \mathrm{s}$. The pressure measurement was done using BMP180- Barometric Pressure Sensor. The magnitude of pressure is electronically recorded using a microcontroller at corresponding points where velocity is measured. After, all necessary velocity vectors were found, the time averaged quantities such as mean velocity, turbulent-velocity fluctuations, normalized turbulent kinetic energy, and Reynolds stress distributions can be numerically simulated using Reynolds averaged Navier Stokes equation.

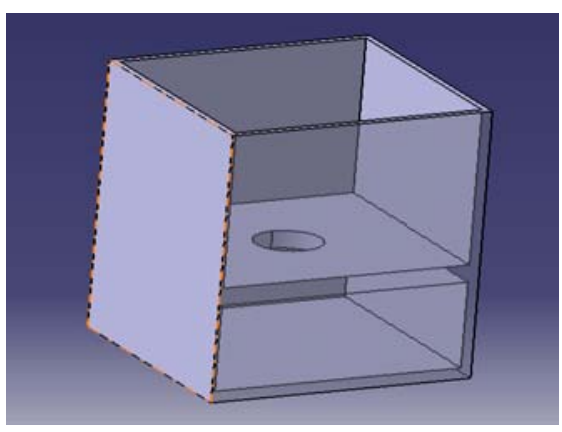

Fig.4 Experimental setup. 


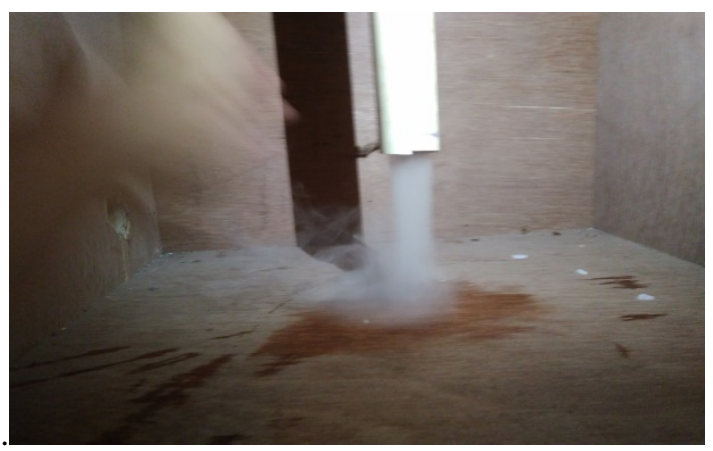

Fig.5 Contact Stage of microburst

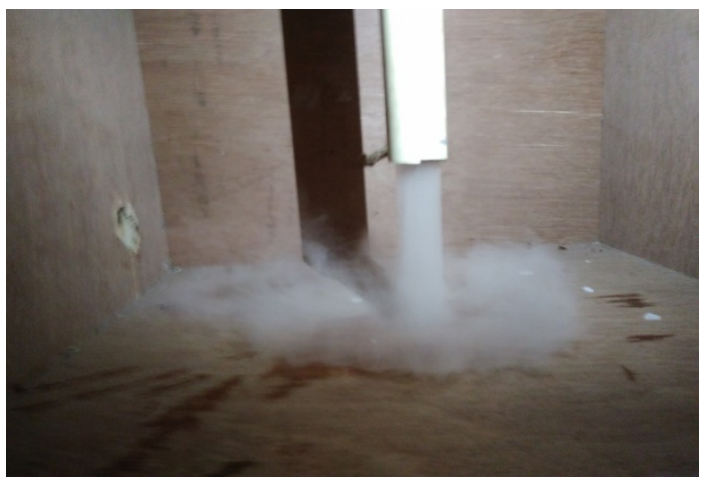

Fig.6. Impinging on ground.

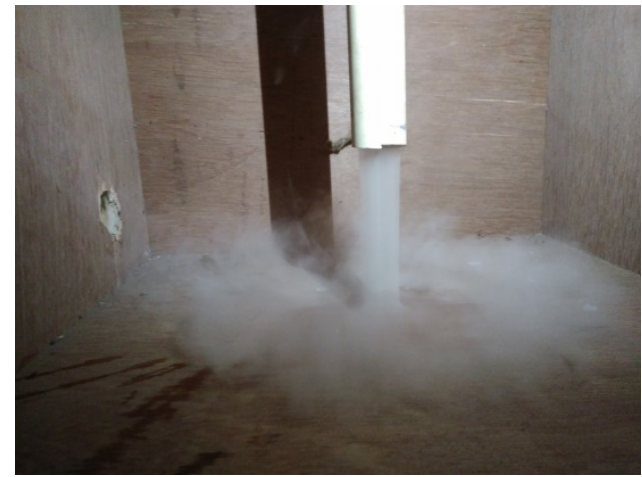

Fig.7 Outburst of microburst

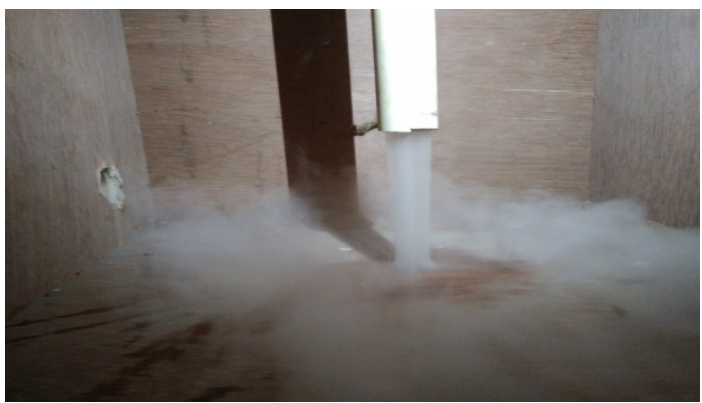

Fig. 8 Cushion stage of microburst

\section{RESUlts AND Discussion:}

In the present study, flow characteristics of microburst like wind generated by blower were quantified by using a Barometric pressure sensor and anemometer. We have taken around 15 measurements for each nozzle diameter of $\mathrm{D}=2.54 \mathrm{~cm}$ and $5.08 \mathrm{~cm}$. The below figures shows flow field in terms of the flow field velocity, the contours maps of vertical velocity component. As a result, microburst like wind was found to have strong vertical components in core region and leading to edge of outburst.

Following graphs are obtained as the microburst model is scaled down to nearly 1:20000 without change in ratio of its relative characteristics. 


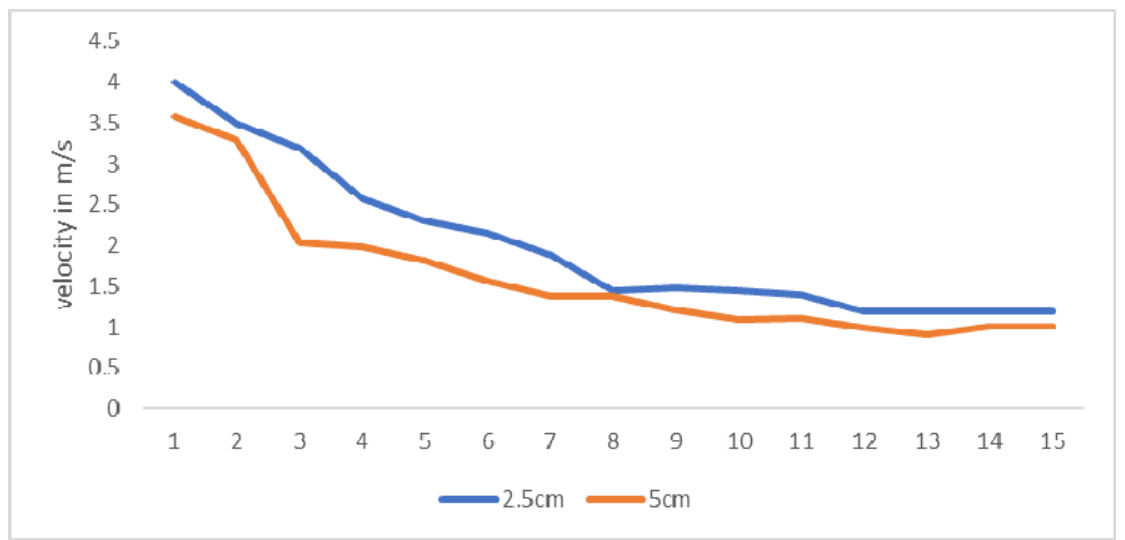

Fig.9. Variation of velocity with radial distance at various heights.

Relationship between the velocity and radial distance from the centre of impingement. As the radial distance increases the velocity reduces due to the fact that in most real vortices, the fluid flow velocity is greatest next to its axis and reduces in inverse proportion to the distance from the axis, here the vortex is formed near $2.5 \mathrm{~cm}$ and $5.08 \mathrm{~cm}$

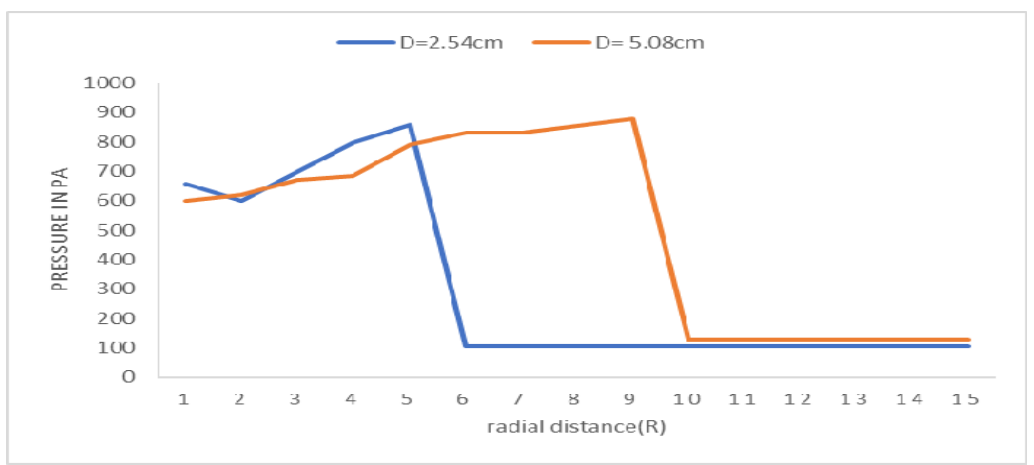

Fig.10. Variation of Pressure with radial distance.

The graph depicts the radial pressure distribution. The pressure which is quite high near the axis of impingement then reduces near $2-2.5 \mathrm{~cm}$ for nozzle diameter $\mathrm{D}=2.54 \mathrm{~cm}$, again increases because the vortex formed by fluid motion creates a dynamic pressure which is lowest in the core region i.e. nearer to the axis, and increases as it moves away from the axis, according to the Bernoulli's Principle. The sudden drop in the pressure at $\mathrm{R}=5 \mathrm{~cm}$ and $\mathrm{D}=2.54 \mathrm{~cm}$ can be explained by the fact that at this point the boundary of the vortex ends.

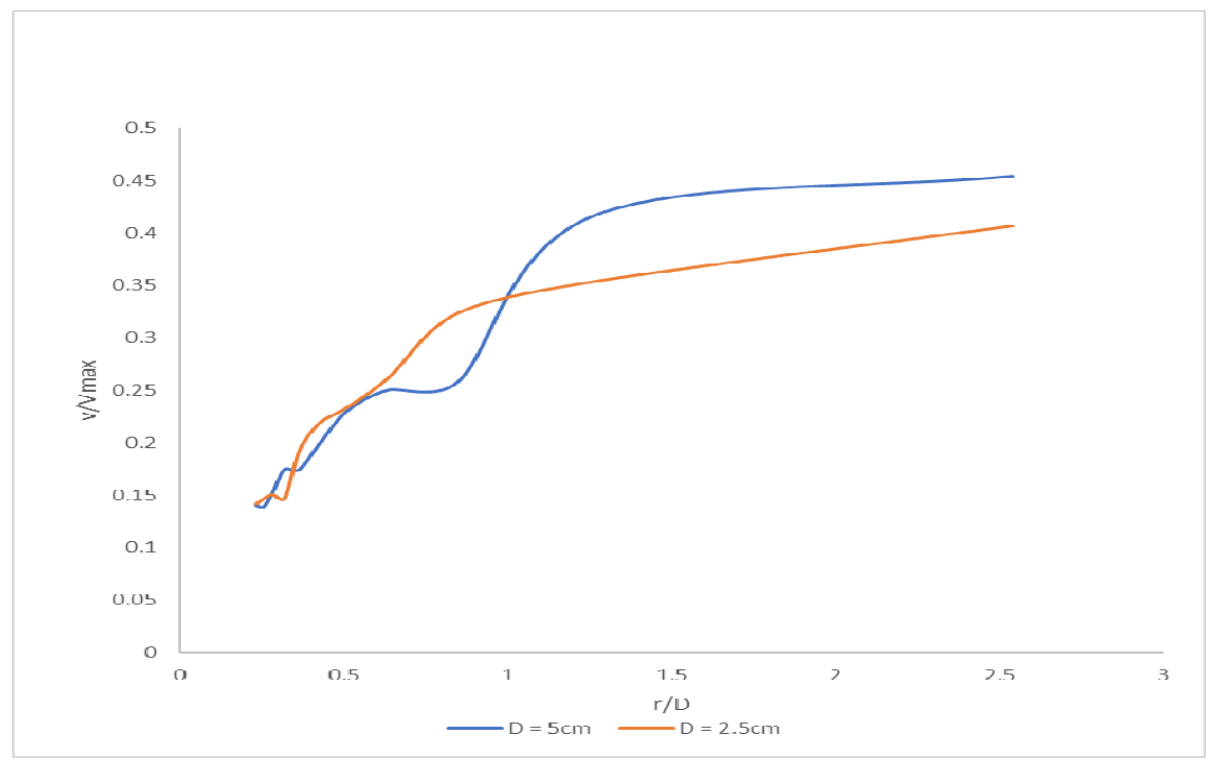

Fig.11. Variation of velocity ratio with radial distance ratio to Diameter of nozzle. 
Dimensionless numbers of velocities are compared so that experiments results can be analysed in scaled model. These variations are very similar to the results obtained in large scale experiment conducted by Iowa state university. This shows the fact that a microburst can be scaled down without change in its velocity profiles.

\section{III.CONCLUSION}

A physical simulator is fabricated for the simulation of the downburst based on the impinging jet model. Radial velocity profiles for different jet velocities and impinging height are investigated. Simulated downburst when compared with the results from the empirical relation of Holmes and Oliver (2000), satisfactory agreement is observed between these results. Smoke is generated using dry ice to visualize the macro flow dynamics of the simulated downburst as shown in fig.5 to fig.8.

The present study reveals the following facts regarding the downburst flow field, maximum radial velocity of the microburst near the ground increases with the increase in jet velocity and decreases to a point in radial direction gradually, the region of high radial velocity moves closer to the impinging plate as the plate separation decreases and microburst flow field characteristics depend on jet velocity, plate separation and jet diameter (nozzle diameter).

\section{REFERENCES}

[1] Fujita T.T., Spearhead echo and downburst near the approach end of a John F. Kennedy runway, New York City, SMRP Research Papers, Univ. of Chicago, 1976.

[2] Fujita T.T., Objective, operations and results of projects NIMROD. Preprint, $11^{\text {th }}$ conference on Severe Local Storms, Amer Meteor Society, Kansas City, 1979.

[3] Fujita T.T., Wakimoto R.M., Five scales of air flow associated with a series of downbursts, Monthly Weather Review, 1980.

[4] Fujita T.T., The downburst, microburst and macroburst. SMRP Res. Paper No.210 [NTIS No. P885-148880] Univ. of Chicago, 1985.

[5] Lundgren TS, Yao J, Mansour NN. Microburst modelling and scaling. J. Fluid Mech, 1992.

[6] Letchford C.W.,Chay M.T., Pressure distributions on a cube in a simulated thunderstorm downburst, 2002.

[7] Holmes JD, Oliver SE. An empirical model of a downburst. Engineering Structures,2000; 22:1167-1172.

[8] Sengupta and Sarkar performed physical modelling to study the flow field characteristics and pressure distribution the stationary and translational downburst, 2007

[9] Sengupta and Sarkar, Experimental simulation and numerical simulation of an impinging jet with application to thunderstorm by turbulence model using FLUENT software, 2008.

[10] Das K.K, Ghosh A.K, Sinhamahapatra K.P,2010. Investigation of axisymmetric microburst flow field. J.Wind and Eng,2010

[11] Gao Zhenxing., Gu Hongbin., Liu Hui., Real time simulation of Large Aircraft Flying through Microburst wind field, 2008.

\section{AUTHOR PROFILE}

D.K. Ramesh, Associate Professor, University Visvesvaraya College of Engineering, Bangalore, Karnataka, India

Gangadhar K A, U.G Scholar, University Visvesvaraya College of Engineering, Bangalore, Karnataka, India

Dhananjaya D R, U.G Scholar, University Visvesvaraya College of Engineering, Bangalore, Karnataka, India

Gokul S, U.G Scholar, University Visvesvaraya College of Engineering, Bangalore, Karnataka, India

Varun Kumar S, U.G Scholar, University Visvesvaraya College of Engineering, Bangalore, Karnataka, India 